8. Kobayashi, S., et al. (2018), "Arthroscopic Treatment of Tibial Eminence Avulsion Fracture With Suture Tensioning Technique", Arthrosc Tech. 7(3), pp. e251-e256.
9. Meyers, M. H. and Mc, Keever Fm (1959), "Fracture of the intercondylar eminence of the tibia", J Bone Joint Surg Am. 41-a(2), pp. 209-20; discussion 220-2.

\title{
MộT Số YẾU TỐ ẢNH HƯởNG TỚI HOẠT ĐộNG NGHIÊN CỨU KHOA HỌC TẠI VIÊ̂N HUYÊT HỌC - TRUYỀN MÁU TRUNG ƯƠNG
}

\section{TÓM TẮT.}

Mục tiêu: Mô tả một sỗ yễu tố ảnh hưởng tới hoạt động nighiên cứu khoa học tại Viện Huyết học - Truyền máu Trung ương giai đoạn 2016-2020. Đối tượng và phương pháp: Nghiên cứu từ tháng 4-10/2021 với thiết kế mô tả cắt ngang, kết hợp định lượng và định tính. Đối tượng gồm 26 cán bộ nhân viên tham gia phỏng vấn và 425 đề tài, 280 bài báo khoa hoc của Viênn từ 2016-2020. Kêt quả: Có 425 đề tài được thực hiện tại Viện, có sự khác biệt về hoạt động NCKH giữa các khối, các trình độ học vấn và chuyên ngành đào tao. Khối lâm sàng có số đề tài, bài báo và tỳ lệ nhân viên tham gia NCKH cao nhất. Tỷ lệ chủ nhiệm đề tài và tác giả bài báo cao nhất ở đối tương bác sĩ $(31 \%$ và $31,6 \%)$, thấp nhất ở đối tượng điều dướng $(7,8 \%$ và $3,2 \%)$. Quan điểm, chiến lược, chính sách khuyến khích và kinh phí hỗ trợ $\mathrm{NCKH}$ của viện, vai trò của lãnh đao khoa phòng, trình độ học vấn cao là các yếu tố cơ bản thúc đẩy NCKH tại viện. Kết luận: Quản lý, điều hành, tài chính và nhân lực là các yếu tố ảnh hưởng tới hoat động NCKH tại Viện.

Từ khoá: Hoạt động NCKH, yếu tố ảnh hưởng, Viện Huyết học - Truyền máu Trung ương.

\section{SUMMARY}

\section{SOME FACTORS AFFECTING SCIENTIFIC} RESEARCH ACTIVITIES AT THE NATIONAL INSTITUTE OF HEMATOLOGY AND BLOOD TRANSFUSION

Objective: Describe some factors affecting scientific research activities at the National Institute of Hematology and Blood Transfusion (NIHBT) for the period 2016-2020. Subjects and methods: Applying cross-sectional design, combining quantitative and qualitative. This study implemented from April to October 2021. The subjects included 26 staff members who participated in interviews and 425 topics and 280 scientific articles of the NIHBT from 2016-2020. Results: There are 425 projects carried out at the NIHBT, there are differences in scientific research

*Viện Huyết học - Truyền máu Trung ương

**Trướng Đai hoc Y tế công công

Chịu trách nhiệm chính: Ngô Huy Minh

Email: huyminh162@gmail.com

Ngày nhận bài: 3.9.2021

Ngày phản biên khoa hoc: 26.10.2021

Ngày duyệt bài: 5.11.2021
Ngô Huy Minh*, Bạch Quốc Khánh*, Nguyễn Hà Thanh*, Vũ Thị Hoàng Lan**

activities between blocks, educational levels and training majors. The clinical division has the highest number of topics, articles and the percentage of staff participating in scientific research. The percentage of topic leaders and article authors was highest among doctors (31\% and $31.6 \%$ ), the lowest among nurses $(7.8 \%$ and $3.2 \%)$. The NIHBT's viewpoints, strategies, policies to encourage and support scientific research, the role of faculty leaders, and high educational attainment are the basic factors promoting scientific research at the NIHBT. Conclusion: Management, administration, finance and human resources are factors affecting scientific research activities at the NIHBT.

Keywords: Scientific research activities, National Institute of Hematology - Blood Transfusion.

\section{I. ĐẶT VẤN ĐỀ}

Nghiên cứu khoa học (NCKH) luôn được coi là hoạt động mang tính then chốt và là một trong bảy nhiệm vụ chính của các bệnh viện. Hàng năm, Viện Huyết học - Truyền máu Trung ương có khoảng 60 đề tài NCKH các cấp về chuyên môn và quản lý được phê duyệt, nghiệm thu và áp dụng thực tế. Tuy nhiên, có sự khác biêt về tỷ lệ tham gia NCKH giữa các khối, khoa phòng và đối tượng. Do đó, chúng tôi thực hiện đề tài với mục tiêu: Mô tả môt số yếu tố ảnh hưởng hoạt động nghiên cứu khoa học tại Viện Huyết họ Truyền máu Trung ương giai đoạn 2016-2020.

II. ĐỐI TƯỢNG VÀ PHƯƠNG PHÁP NGHIÊN CỨU

\section{1. Đối tượng nghiên cứu:}

- Đinh tính: 26 người bao gồm đại diên lãnh đạo Viện, các khoa phòng liên quan, viên chức của Viện.

- Định lượng: 425 đề tài, 280 bài báo của Viện giai đoạn 2016-2020.

2.2. Thiết kế nghiên cứu: Mô tả cắt ngang kết hợp định lượng và định tính.

2.3. Phương pháp chọn mẫu và cỡ mẫu: Chon chủ đích 26 đối tượng nghiên cứu định tính: Lãnh đạo viện, bác sĩ, điêu dưỡng, kỹ thuật viên và đối tượng ngoài ngành $y$, chủ nhiệm đề tài, tác giả bài báo, đối tượng không tham gia $\mathrm{NCKH}$....và các đề tài, bài báo liên quan. 
2.4. Công cụ và phương pháp thu thập, xử lý số liệu: Sử dụng phiếu thu thập thông tin để tổng hợp kết quả từ các đề tài, bài báo. Sử dụng máy ghi âm/sổ ghi chép để tìm hiểu các yếu tố ảnh hưởng theo khung hệ thống y tế rút gọn. Xử lý số liệu bằng phần mềm SPSS 20.0 với các test thống kề X2, T- Test... gõ băng, trích dẫn.

\section{KẾT QUẢ NGHIÊN CỨU}

3.1. Kết quả thực hiện các đề tài, bài báo NCKH giai đoạn 2016-2020

Bảng 3.1: Số đề tài bài báo, khoa hoc được quản lý

\begin{tabular}{|c|c|c|c|c|c|c|c|c|}
\hline \multirow[b]{2}{*}{ Nôi dung } & \multicolumn{2}{|c|}{ Cấp Cơ sở } & \multicolumn{2}{|c|}{ Cấp Bô, Quốc gia } & \multicolumn{2}{|c|}{ Hơp tác } & \multicolumn{2}{|c|}{ Tống } \\
\hline & $\mathbf{n}$ & $\%$ & n & $\%$ & $\mathbf{n}$ & $\%$ & $\mathbf{n}$ & $\%$ \\
\hline Tống đề tài quản lý & 411 & 96,7 & 2 & 0,5 & 12 & 2,8 & 425 & 100 \\
\hline Đề tài phê duyệt & 381 & 96,5 & 2 & 0,5 & 12 & 3,0 & 395 & 100 \\
\hline Đề tài nghiệm thu & 255 & 97,3 & 1 & 0,4 & 6 & 2,3 & 262 & 100 \\
\hline \multirow{3}{*}{ Số bài báo công bố } & \multicolumn{3}{|c|}{ Tạp chí trong nước } & \multicolumn{3}{|c|}{ Tạp chí quốc tế } & \multicolumn{2}{|c|}{ Tống } \\
\hline & \multirow{2}{*}{\multicolumn{2}{|c|}{$\frac{\mathbf{n}}{268}$}} & $\%$ & $\mathrm{n}$ & \multirow{2}{*}{\multicolumn{2}{|c|}{4,3}} & $\mathbf{n}$ & $\%$ \\
\hline & & & 95,7 & 12 & & & 280 & 100 \\
\hline
\end{tabular}

Nhân xét: Tống số Viên quản lý 425 đề tài, tỷ lệ đề tài cấp cơ sở chiếm $96,7 \%$, có 12 đề tài hợp tác với các đơn vị khác. Hầu hết bài báo đăng tại các tạp chí trong nước. Có 12 bài báo được đăng tại các tạp chí quốc tế.

Bảng 3.2: Số lượng đề tài NCKH được phê duyệt và nghiệm thu theo khối

\begin{tabular}{|c|c|c|c|c|c|c|c|c|c|c|c|c|}
\hline \multirow{4}{*}{ Nội dung } & \multirow{2}{*}{\multicolumn{4}{|c|}{ Huyết học }} & \multirow{3}{*}{\multicolumn{2}{|c|}{$\begin{array}{l}\text { Truyền } \\
\text { máu }\end{array}$}} & \multirow{3}{*}{\multicolumn{2}{|c|}{$\begin{array}{l}\text { Hành } \\
\text { chính }\end{array}$}} & \multirow{3}{*}{\multicolumn{2}{|c|}{ Tổng }} & \multirow{4}{*}{$\begin{array}{c}\begin{array}{c}\text { Kế } \\
\text { hoạch }\end{array} \\
\mathbf{n}\end{array}$} & \multirow{4}{*}{ 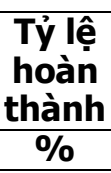 } \\
\hline & & & & & & & & & & & & \\
\hline & \multicolumn{2}{|c|}{ Lâm sàng } & \multicolumn{2}{|c|}{$\begin{array}{c}\text { Cận } \\
\text { lâm sàng }\end{array}$} & & & & & & & & \\
\hline & II & $\%$ & $n$ & $\%$ & 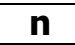 & $\%$ & $\mathbf{n}$ & $\%$ & $\mathrm{n}$ & $\%$ & & \\
\hline Phê duyệt & 156 & 39,5 & 78 & 19,7 & 130 & 32,9 & 31 & 7,9 & 395 & 100 & 175 & 226 \\
\hline Nghiệm thu & 88 & 33,6 & 67 & 25,6 & 92 & 35,1 & 15 & 5,7 & 262 & 100 & 150 & 175 \\
\hline
\end{tabular}

Nhận xét: Viện hoàn thành vượt mức trên $100 \%$ kề hoạch phê duyệt đề tài NCKH. Tỷ lê đề tài được phê duyệt ở khối lâm sàng cao nhất trong toàn Viện (39,5\%). Tỷ lệ nghiệm thu đề tài đạt $175 \%$ kế hoạch. Khối truyền máu và lâm sàng có tỷ lệ nghiệm thu cao nhất.

3.2. Một số yếu tố ảnh hưởng tới hoạt động NCKH tại Viện

\subsubsection{Các yếu tố cá nhân}

Bảng 3.3: Tỷ lệ tham gia nghiên cứu khoa học theo nhóm tuổi và khôi làm việc

\begin{tabular}{|c|c|c|c|c|c|c|c|}
\hline \multirow{2}{*}{ Nội dung } & \multicolumn{2}{|c|}{ Tham gia } & \multicolumn{2}{|c|}{ Không tham gia } & \multicolumn{2}{|c|}{ Tống } & \multirow{2}{*}{ Po } \\
\cline { 2 - 7 } & $\mathbf{n}$ & $\mathbf{\%}$ & $\mathbf{n}$ & $\mathbf{\%}$ & $\mathbf{n}$ & $\mathbf{\%}$ & $\mathbf{P}$ \\
\hline Nhóm tuối <30 (1) & 119 & 32,9 & 243 & 67,1 & 362 & 42,2 & $\mathrm{p} 1,2<0,05$ \\
$30-45(2)$ & 287 & 68,2 & 134 & 31,8 & 421 & 49,1 & $\mathrm{p} 1,3<0,05$ \\
$>45(3)$ & 48 & 64,0 & 27 & 36,0 & 75 & 8,7 & $\mathrm{p} 2,3>0,05$ \\
Tổng & 454 & 52,9 & 404 & 47,1 & 858 & 100 & \\
\hline Khối làm việc & & & & & & & \\
Huyết học (1) & 278 & 62,1 & 170 & 37,9 & 448 & 52,2 & $\mathrm{p} 1,2<0,05$ \\
Truyền máu (2) & 120 & 43,3 & 157 & 56,7 & 277 & 32,3 & $\mathrm{p} 1,3<0,05$ \\
Hành chính (3) & 56 & 42,1 & 77 & 57,9 & 133 & 15,5 & $\mathrm{p} 2,3>0,05$ \\
\hline
\end{tabular}

Nhận xét: Tỳ lệ tham gia NCKH chiếm được cần nghiên cứu về cái gi và họ cũng có $52,9 \%$ tổng số nhân lực toàn viện. Nhóm tuổi chủ yếu là từ 30-45. Khối huyết học tham gia NCKH cao nhất.

Một số kêt quả thảo luận nhóm: "Tuổi trẻ ít đam mê làm đề tài hơn, tầm trung tuổi có trải nghiệm nhiều trong công việc nên có thể nghĩ nhiều kiến thức hơn" (TLN1)

"Khoa phòng làm việc và đặc thù nghề nghiệp được đào tạo có ảnh hưởng tới việc tìm kiếm để tài nghiên cứu, có khoa có rất nhiều chủ đề nghiên cứu, có khoa tìm rất khó" (TLN1, 4),

"Như em ở khoa lâm sàng H6, hiện tại vẫn có thể giành thời gian tham gia NCKH được" (TLN5)

Bảng 3.4: Tỷ lệ chủ nhiệm đề tài và tác giả bài báo theo chuyên ngành đào tạo

\begin{tabular}{|c|c|c|c|c|c|c|c|}
\hline \multirow{2}{*}{$\begin{array}{c}\text { Chuyên ngành } \\
\text { đào tạo }\end{array}$} & \multicolumn{2}{|c|}{ Chủ nhiệm } & \multicolumn{2}{|c|}{ Tác giả bài báo } & \multicolumn{2}{|c|}{ Tống nhân lực } & \multirow[b]{2}{*}{$\mathbf{p}$} \\
\hline & $\mathbf{n}$ & $\%$ & $\mathrm{n}$ & $\%$ & $\mathbf{n}$ & $\%$ & \\
\hline Bác sĩ, dược sĩ (1) & 53 & 31,0 & 54 & 31,6 & 171 & 19,9 & p $1(2,3,4)<$ \\
\hline Điều dưỡng (2) & 29 & 7,8 & 12 & 3,2 & 370 & 43,1 & 0,05 \\
\hline
\end{tabular}




\begin{tabular}{|c|c|c|c|c|c|c|c|}
\hline Kỹ thuật viên (3) & 28 & 16,4 & 14 & 8,2 & 171 & 19,9 & $\mathrm{p} 2(3,4)<0,05$ \\
\cline { 1 - 5 } Ngoài ngành y (4) & 27 & 18,5 & 20 & 13,7 & 146 & 17,0 & p $3(1,2)<0,05$ \\
\cline { 1 - 5 } Tống & $\mathbf{1 3 7}$ & $\mathbf{1 6 , 0}$ & $\mathbf{1 0 0}$ & $\mathbf{1 1 , 7}$ & $\mathbf{8 5 8}$ & $\mathbf{1 0 0}$ & p $3,4>0,05$ \\
\hline
\end{tabular}

Nhận xét: Bác sĩ, dược sĩ có tỷ lệ làm CNĐT và tác giả bài báo cao nhất. Điều dưỡng là đối tượng có tỷ lệ làm CNĐT và tác giả bài báo thấp nhất.

"Nhân viên của viện trẻ, đặc biệt là ĐD- KTV, vấn đề là họ chưa được học về NCKH trong quá trình đào tạo" (PVS2)

Bảng 3.5: Năng suât NCKH của chủ nhiệm đề tài và tác giả bài báo

\begin{tabular}{|c|c|c|c|c|c|c|c|}
\hline \multirow{3}{*}{ Trình độ học vấn } & \multirow{3}{*}{$\mathbf{n}$} & \multicolumn{6}{|c|}{ Năng suất trung bình 5 năm } \\
\hline & & \multicolumn{3}{|c|}{ Chủ nhiệm đêê tài } & \multicolumn{3}{|c|}{ Tác giả bài báo } \\
\hline & & Nam & Nũ & Chung & Nam & $\mathbf{N} \tilde{u}$ & Chung \\
\hline GS, PGS, TS, BSCKII (1) & 27 & 6,1 & 3,2 & 4,8 & 4,9 & 5,5 & 5,1 \\
\hline Thạc sĩ, DS, BSCKI (2) & 48 & 1,7 & 1,8 & 1,8 & 1,6 & 2,6 & 2,1 \\
\hline Bác sĩ, cử nhân ĐH (3) & 38 & 0,9 & 0,8 & 0,8 & 0,7 & 0,9 & 0,8 \\
\hline Dưới đại học (4) & 24 & 0,6 & 0,8 & 0,7 & 0,1 & 0,4 & 0,3 \\
\hline Trung bình 5 năm & 137 & 2,4 & 1,6 & 1,9 & 2,0 & 2,1 & 2,0 \\
\hline \multirow{2}{*}{\multicolumn{2}{|c|}{$p$}} & \multicolumn{3}{|c|}{$p<0,05$} & \multicolumn{3}{|c|}{$p>0,05$} \\
\hline & & \multicolumn{3}{|c|}{$\begin{array}{c}\text { p } 1(2,3,4)<0,05 \\
\text { p } 2(3,4)<0,05 ; \text { p } 3,4>0,05\end{array}$} & \multicolumn{3}{|c|}{ p $1,2,3,4<0,05$} \\
\hline
\end{tabular}

Nhân xét: Có sự khác biệt giữa các đối tượng có trình độ học vấn khác nhau và giới đối với năng suất làm CNĐT và tác giả bài báo.

"Trình độ học vấn có tác động đến việc NCKH do việc tiếp cận với vấn đề nhiều và rộng hơn nên có nhiêuu cơ hội để nghiên cứu" (TLN6)

"Phụ nữ còn cống việc gia đình, con nhỏ nên có thể ảnh hưởng tới NCKH" (TLN4, 5)

3.2.2. Các yếu tố quản trị, điêuu hành. "Nghiên cứu khoa học là mối quan tâm hàng đâu, là một ưu tiên phát triển của viện vì đây là công việc then chốt của viện đâu ngành về HHTM trên toàn quốc... ngoài việc tập trung vào các lĩnh vực chuyên môn chư chốt của viện, chúng tôi cũng nhấn mạnh tới các lĩnh vực nghiên cứu về quản lý như vận động hiến máu tình nguyện, tiếp thị xã hội"(PVS1).

"Cần nghiên cứu tất cả các lĩnh vực, ưu tiên các vấn đề mang tính mũi nhọn và nên có các cấp độ nghiên cứu khác nhau" (TLN1).

"Các chính sách khuyến khích như khen thưởng, xét thi đua, nâng lương sớm là yếu tố thúc đây, là động lực để anh, chị, em tham gia nhiều đề tài NCKH hơn" (TLN5).

"Sự hỗ trợ của lãnh đạo khoa phòng là rất lớn để ĐD-KTV tham gia NCKH từ lựa chọn vấn đề, phân tích số liệu, trình bày bài báo và cân được tăng cường" (TLN9).

3.2.3. Yếu tố tài chính. "Yếu tố tài chính là vô cùng quan trợng và cân thiết, trong thời gian qua viện đã hỗ trợ miễn phí đăng các bài báo trên các tạp chí, hàng năm đều dành kinh phí cho công tác đào tạo về NCKH và thực hiện đề tài và đã góp phân khuyến khích CBNV tham gia NCKH" (PVS1).
"Kinh phí được cấp cho các đề tài đủ để thực hiện nghiên cưuu, tuy nhiên, chưa có kinh phí khen thưởng với những bài đăng quốc tế..."(PVS3).

"Nguồn kinh phí để thực hiện cho NCKH còn hạn hẹp là những yếu tố khó khăn trong quá trình NCKH của các bác sỹ tại viện" (TLN3).

\section{BÀN LUẬN}

Viện đã hoàn thành vượt mức kế hoạch NCKH trong 5 năm với 395 đề tài được phê duyệt, 262 đề tài nghiệm thu và 280 bài báo được công bố, nhiều đề tài hợp tác được triển khai. 454 cán bộ nhân viên đã tham gia NCKH chiếm tỷ lệ 52,9\% nhân lực toàn viện (bảng 3.1, 3.2.). Tỷ lệ tham gia NCKH tương đối cao nhưng có sự khác biệt giữa các khối, đối tượng tham gia NCKH. Dưới đây là một số yếu tố ảnh hưởng chính đến hoạt động NCKH của Viện.

4.1. Các yếu tố cá nhân: Nhóm tuổi là yếu tố ảnh hưởng tới việc tham gia $\mathrm{NCKH}$, các đối tượng < 30 tuổi tham gia NCKH ít hơn nhóm tuổi từ 30 trở lên. Sự khác biệt có ý nghĩa thống kê với $p<0,05$. Các ý kiến thảo luận nhóm cũng cho rằng ở nhóm tuổi dưới 30 còn thiếu kiến thức, kinh nghiệm và đam mê nghiên cứu.

Khoa phòng làm việc được đánh giá ảnh hưởng tới hoạt động NCKH thông qua gánh nặng công việc và cơ hội tìm kiếm đề tài nghiên cứu. Khối huyết học là đơn vị tiên phong với tỷ lệ phê duyệt đề tài và tỷ lệ nhân lực tham gia NCKH cao nhất trong các khối (bảng 3.2, 3.3). Theo ý kiến thảo luận nhóm, mặc dù công việc của khối tương đối bận nhưng vẫn có thể sắp xếp được thời gian NCKH. Số lượng nhân lực có trình độ cao và nhiều chủ đề nghiên cứu là các yếu tố 
giúp khối huyết học luôn dẫn đầu trong hoạt động $\mathrm{NCKH}$. Đa số ý kiến khối truyền máu và khối hành chính thống nhất rằng cơ hội tìm kiếm chủ đề phụ thuộc vào từng khoa phòng công tác. Mặc dù đặc thù của khối truyền máu là phải lưu động nhiều nhưng vẫn có thể dành thời gian cho NCKH. Kết quả của chúng tôi có khác biêt với nghiên cứu của Hà Hữu Tùng [1], Đoàn Thi Ngần [2] khi cho rằng các CNĐT không có thời gian lấy số liệu, phân tích, viết báo cáo kết quả do bận công tác chuyên môn. Như vậy có thể thấy Viên đã làm tốt công tác nhân lực, ngoài việc thực hiện nhiệm vụ chuyên môn thì CBNV vẫn sắp xếp được thời gian cho NCKH. Yếu tố ảnh hưởng chủ yếu là cơ hội tìm kiếm chủ đề nghiên cứu ở mỗi khoa phòng khác nhau.

Có sự khác biệt có ý nghĩa thống kê trong NCKH giữa các chuyên ngành đào tạo: Tỷ lệ làm chủ nhiệm đề tài (CNĐT) và tác giả bài báo cao nhất ở đối tượng bác sỹ ( $31 \%$ và $31,6 \%)$ và thấp nhất ở đối tượng điều dưỡng $(7,8 \%$ và $3,2 \%)$. Các kết quả nghiên cứu định tính cho thấy môt số nguyên nhân dẫn đến tỷ lệ điều dưỡng tham gia NCKH thấp hơn các đối tượng khác là do thiếu kiến thức về NCKH, không được học về nội dung này trong quá trình đào tạo nển rẩt khó khăn trong việc tự tìm kiếm đề tài và triển khai nghiên cứu (bảng 3.4).

Kết quả tại bảng 3.5 cho thấy có sự khác biêt về năng suất $\mathrm{NCKH}$ giữa các trình độ học vẩn (TĐHV) khác nhau. Các đối tượng từ BSCKII trở lên có năng suất $\mathrm{NCKH}$ là 4,8 đề tài, 5,1 bài báo/5 năm, cao nhất trong các đối tượng. Các đối tượng trình độ đại học và thạc sĩ có năng suất NCKH thấp hơn nhiều so với các đối tượng từ BSCKII trở lên. Kết quả này tương đồng với nghiên cứu của Huỳnh Trường Huy khi cho rằng TĐHV cao thì số lượng và chất lượng các ấn phẩm khoa học được xuất bản cao hơn [3]. Các ý kiến thảo luận nhóm cũng cho thấy TĐHV là một yếu tố quan trọng ảnh hưởng tới việc tham gia cũng như năng suất NCKH. Các nhân viên trẻ, các đối tượng điều dưỡng có TĐHV chưa cao nên thiếu kiến thức và không tự tin tham gia NCKH.

Năng suất làm CNĐT của nam cao hơn nữ ( 2,4 và 1,6 đề tài).Việc đứng tên là CNDT sẽ chiếm nhiều thời gian của nghiên cứu viên hơn khi chỉ là thành viên. Các ý kiến thảo luận nhóm cho thấy phụ nữ có nhiều yếu tố vướng bận gia đình hơn nam giới nên sẽ ảnh hưởng tới việc chủ trì đề tài NCKH. Kết quả của chúng tôi phù hợp với nghiên cứu của Huỳnh Trường Huy, Yue Xie \& Shauman $[3,4]$.

4.2. Yếu tố quản trị, điêu hành. Về quan điểm, lãnh đạo Viện đã xác định NCKH là mối quan tâm hàng đầu cần phải ưu tiên thực hiện vì đây là nhiêm vụ then chốt của viện đầu ngành. Viện đã thống nhất triển khai nghiển cứu trền tất cả các lĩnh vực, khoa phòng với nhiều loại hình nghiên cứu khác nhau. Viện đã có các chính sách khuyến khích NCKH cụ thể, thực chất và hiệu quả cả về tinh thần và vật chất thông qua các tiêu chuẩn thi đua, nâng lương sớm cũng như chính sách hỗ trợ kinh phí thực hiện đề tài, đăng báo, kinh phí tham dự hội nghị, hội thảo. Hầu hết các ý kiến đều hài lòng với những chính sách trên và cho rằng đó là động lực để họ tham gia $\mathrm{NCKH}$. Kết quả của chúng tôi cũng tương đồng với nghiên cứu của Hà Hữu Tùng, Trần Thị Kim Nhung $[1,5]$.

Sự hố trợ của lãnh đạo khoa phòng: Kết quả nghiển cứu cho thấy các ý kiến đều đánh giá cao sự hỗ trợ của lãnh đạo khoa phòng từ sắp xếp cổng việc, gợi ý các vấn đề nghiên cứu đến phân tích và trình bày số liệu. Tuy nhiên, sự hố trợ này mới chỉ được thực hiện tại một số khoa phòng và các ý kiến đểu mong muốn nhận được sự trợ giúp nhiều hơn nữa từ lãnh đạo khoa phòng. Kết quả này cũng phù hợp với nghiên cứu của Đoàn Thị Ngần [2].

4.3. Yếu tố tài chính. Đại diện lãnh đạo viện khẳng định yếu tố tài chính là vồ cùng quan trọng và cần thiết và viện sẽ tiếp tục duy trì, đẩy mạnh công tác này để thúc đẩy hoạt động $\mathrm{NCKH}$ tại viện. Các ý kiến thảo luận nhóm cũng cho rằng sự hỗ trợ về kinh phí góp phần động viên mọi người tham gia đề tài, đăng bài báo và đa số hài lòng với mức hỗ trợ của viện. Một số ý kiến cho rằng trong giai đoạn 2016- 2020 có thời điểm kinh phí hỗ trợ cho đề tài thấp, là yếu tố khó khăn trong thực hiện NCKH. Chúng tôi đã tìm hiểu và thấy rằng tại thời điểm đó, do số lượng đề tài được phể duyệt rất nhiều nên kinh phí cho mỗi đề tài có giảm hơon và vấn đề này đã được khắc phục ở những năm tiếp theo. Như vậy có thể thấy, tài chính là một yếu tố ảnh hưởng đến hoạt động $\mathrm{NCKH}$ của viện. Kết quả của chúng tồi cũng tương đồng với nghiên cứu của Hà Hữu Tùng, Trần Thị Kim Nhung khi cho thấy yếu tố tài chính và sự hố trợ của tổ chức có ảnh hưởng lớn tới NCKH $[1,5]$.

\section{KẾT LUẬN}

Quan điểm, chiến lược và chính sách khuyến khích NCKH, sự hỗ trợ về kinh phí của lãnh đạo viện là các yếu tố quan trọng thúc đẩy hoạt động NCKH tại Viện. Sự hỗ trợ của lãnh đạo khoa phòng, trình độ học vấn từ sau đại học, nhóm tuổi 
Z30 và giới nam, khoa phòng làm việc có nhiêu chủ đề, lĩnh vực nghiên cứu là yếu tố cơ bản góp phân nâng cao tỷ lệ tham gia NCKH tại Viện. Đối tượng điêuu dưỡng, trình độ dưới đại học, thiếu kiến thức về NCKH, nhóm tuổi dưới 30 , giới nữ là các yễu tố chính hạn chễ tham gia NCKH.

\section{TÀI LIÊU THAM KHẢO}

1. Hà Hữu Tùng (2017). Thực trạng hoạt đông nghiên cứu khoa học tại bệnh viện đa khoa Nống nghiệp giai đoạn 2014-2016. Tạp chí Y học Việt Nam, 451:200-i்5.

2. Đoàn Thị Ngân (2018). Thực trạng tham gia nghiên cứu khoa học và nhu câuu đào tạo của điêu dưỡng Bệnh viện Thống nhất năm 2018, trang 27, 39, 40, 56. Luận văn thạc sĩ quản lý bệnh viện, Trường Đai hoc $Y$ tế Công công, Hà Nối.

3. Huỳnh Trườing Huy và cộng sự (2015). Phân tích năng suất nghiên cứu khoa học của giảng viên nữ tại Trường Đai học Cần Thơ. Tạp chí khoa học trường Đai học Cần Tihơ, (36):81-9i.

4. Xie, Y. và K. A. Shauman (1998). Sex differences in research productivity: New evidence about an old puzzle. American Sociological Review, 63:847-870.

5. Trân Thị Kim Nhung (2020). Các yếu tố ảnh hưởng tới động lực nghiên cứu khoa học của giảng viên đai hoc khối kinh tế tai Hà Nôi, trang 122. Luận án tiến sĩ ngành quản lý kinh tế, Hà Nội.

\section{KẾT QUẢ ĐIỀU TRỊ UNG THƯ PHỔI BIỂU MÔ TUYẾN DI CĂN NÃO CÓ ĐộT BIẾN EGFR BẰNG TKIs THẾ HỆ 1}

\section{TÓM TẮT}

Mục tiêu: Đánh giá kết quả điều tri ung thư phổi biểu mô tuyến di căn di căn não có đột biến EGFR bằng TKIs thế hệ 1 và một số yếu tố liên quan đến PFS. Đối tượng nghiên cứu: Gồm 66 bệnh nhân UTP biểu mô tuyến di căn não có đôt biến gen EGFR (Del exon 19 hoặc L858R exon 21) được điêu trị bước 1 bằng Gefitinib $250 \mathrm{mg} / \mathrm{ngày}$ hoăc Erlotinib $150 \mathrm{mg} / \mathrm{ngày}$ có kết hợp với xạ toàn não hoặc gamma knife hoặc không tại bệnh viện $K$ từ tháng 10/2015 đến tháng 1/2021. Kết quả: Trung vị PFS đạt 11,5 tháng. PFS trung vị của các nhóm dùng TKIs đơn thuân, TKIs + xạ toàn não, TKIs + gamma knife lần lượt là 11 tháng, 11 tháng và 12,7 tháng. PFS não trung vị đat 11 tháng. Các yếu tố liên quan đến PFS là có đột biển del 19, đáp ứng tại thời điểm 3 tháng, toàn trang tốt, không hoăc di căn 1 cơ quan ngoài não. Kểt luâan: Điều trị TKIs thế hệ 1 trên bệnh nhân UTP biểu mổ tuyến di căn não mang lại tỷ lệ đáp ứng chung cũng như đáp ứng tại não cao, kéo dài thời gian sống thêm bệnh không tiến triển, kết quả khả quan ở nhóm điều trị kết hợp với gamma knife.

Tư khóa: TKIs thế hệ 1, ung thư phổi di căn não, đột biến EGFR.

\section{SUMMARY \\ RESULT OF TREATMENT FIRST-GENERATION TYROSIN KINASE INHIBITORS IN BRAIN METASTASES FROM ADENOCARCINOMA}

\footnotetext{
${ }^{1}$ Trường Đại học Y Hà Nội

Bênh viên $K$

Chịu trách nhiệm chính: Vũ Huyền Trang

Email: vuhuyentrang.hmu@gmail.com

Ngày nhận bài: 23.8.2021

Ngày phản biên khoa hoc: 25.10.2021

Ngày duyệt bài: 4.11.2021
}

Vũ Huyền Trang ${ }^{1}$, Nguyễn Văn Hiếu ${ }^{1,2}$, Nguyễn Thị Thúy Hằng ${ }^{2}$, Nguyễn Quang Anh ${ }^{1}$

OF THE LUNG WITH EGFR MUTATION

Objectives: Evaluate the result of treatment firstgeneration tyrosine kinase inhibitors in EGFR positive adenocarcinoma of lung with brain metastases and describe some factors related to PFS. Patients and Methods: 66 patients adenocarcinoma of lung have brain metastasis withEGFR mutations (exon 19 deletion and L858R point mutation exon 21) were treated with Gefitinib $250 \mathrm{mg} /$ day or Erlotinib $150 \mathrm{mg} /$ day, either whole brain radiation therapy or gamma knife was included or not. Results: The median PFS achieved 11,5 months. The median PFS of TKIs, TKIs + WBRT, TKIs + SRS were 11,11 and 12,7 months, respectively. The median intracranial PFS was 11 months. Some factors predicts favourable outcomes are exon 19 deletion, response at 3-month and performance stasus $0-1$, non or only one organ extracranial metastases. Conclusion: First-generation TKIs for brain metastases in adenocarcinoma of lung with EGFR mutation improved progression-free survival, especially in group used gamma knife in local therapy.

Keywords: first-generation TKIs, brain metastases in lung cancer, EGFR mutation.

\section{I. ĐĂT VẤN ĐỀ}

Ung thư phổi (UTP) là một trong những bênh ung thư phổ biến nhất trên thế giới. Theo thống kê của Globocan năm 2020, trên thế giới có khoảng 2,2 triệu ca mới mắc UTP, chiếm $11,4 \%$ tổng số ca mới mắc ${ }^{1}$. UTP là loại ung thư gây tử vong hàng đầu ở nam giới, hàng năm có khoảng 1,8 triệu người chết, chiếm tỷ lể $18 \%$ tổng số ca tử vong do ung thư'1. Di căn não là vị trí thường găp thứ 3 sau xương và phổi đối bên ở bênh nhân ung thư phối giai đoạn muộn với tỷ lệ lên đến $28,4 \%{ }^{2}$. Trước đây, di căn não được biết đến là yếu tố tiên lượng xấu, bệnh nhân suy sụp 